\title{
Dental procedures, antibiotic prophylaxis, and endocarditis among people with prosthetic heart valves: nationwide population based cohort and a case crossover study
}

\author{
Sarah Tubiana, ${ }^{1,2}$ Pierre-Olivier Blotière, ${ }^{2}$ Bruno Hoen, ${ }^{3}$ Philippe Lesclous, ${ }^{4}$ Sarah Millot, ${ }^{5}$ \\ Jérémie Rudant, ${ }^{2}$ Alain Weill, ${ }^{2}$ Joel Coste, ${ }^{2}$ François Alla, ${ }^{2}$ Xavier Duval ${ }^{1}$
}

INSERM, IAME, UMR 1137

Paris, France; Université Paris

Diderot, IAME, UMR 1137,

Sorbonne Paris Cité, Paris,

France; INSERM CIC-1425,

Assistance Publique-Hôpitaux

de Paris, Hôpital Bichat Claude

Bernard, Paris, France

${ }^{2}$ Department of Studies in Public Health, French National Health

Insurance, Paris Cedex 20, France

${ }^{3}$ Service de Maladies Infectieuses et Tropicales et Inserm-CIC 1424, Centre Hospitalier Universitaire de Pointe-à-Pitre, Pointe-à-Pitre,

France; Université des Antilles

et de la Guyane, Faculté de

Médecine Hyacinthe Bastaraud,

Pointe-à-Pitre, Guadeloupe,

France

${ }^{4}$ INSERM, U 1229, RMeS, Nantes, France, UFR

d'Odontologie, Université de Nantes, Nantes, France, CHU Hôtel Dieu, Nantes, France

${ }^{5}$ Department of Odontology,

CHRU Université de Montpellier

France; UMR 1149 INSERM, CRI.

Université Paris Diderot, France

Correspondence to: STubiana

sarah.tubiana@aphp.fr

Additional material is published online only. To view please visit the journal online.

Cite this as: $B M J$ 2017;358:j3776 http://dx.doi.org/10.1136/bmj.j3776

Accepted: 02 August 2017

\section{ABSTRACT}

OBJECTIVE

To assess the relation between invasive dental procedures and infective endocarditis associated with oral streptococci among people with prosthetic heart valves.

DESIGN

Nationwide population based cohort and a case crossover study.

\section{SETTING}

French national health insurance administrative data linked with the national hospital discharge database.

\section{PARTICIPANTS}

All adults aged more than 18 years, living in France, with medical procedure codes for positioning or replacement of prosthetic heart valves between July 2008 and July 2014.

\section{MAIN OUTCOME MEASURES}

Oral streptococcal infective endocarditis was identified using primary discharge diagnosis codes. In the cohort study, Poisson regression models were performed to estimate the rate of oral streptococcal infective endocarditis during the three month period after invasive dental procedures compared with non-exposure periods. In the case crossover study, conditional logistic regression models calculated the odds ratio and $95 \%$ confidence intervals comparing exposure to invasive dental procedures during the three month period preceding oral streptococcal infective endocarditis (case period) with three earlier control periods.

\section{WHAT IS ALREADY KNOWN ON THIS TOPIC}

Current data suggest that everyday life bacteraemia (induced by, for example, tooth brushing, chewing) may more likely be responsible for infective endocarditis than bacteraemia induced by invasive dental procedures

Frequency and intensity of everyday life and post-procedure bacteraemia are higher in patients with poor oral hygiene and periodontal diseases

Efficacy of infective endocarditis antibiotic prophylaxis in patients with predisposing cardiac conditions undergoing invasive dental procedures is still debated

\section{WHAT THIS STUDY ADDS}

Incidence of oral streptococcal infective endocarditis in relation to everyday life bacteraemia is 94.6 per 100000 person years (95\% confidence interval 82.5 to 106.6) among patients with prosthetic heart valves

Invasive dental procedures during the three months preceding oral streptococcal infective endocarditis are recorded in only $5.1 \%$ of patients with prosthetic heart valves

Invasive dental procedures may contribute to the development of infective endocarditis in the population of patients with prosthetic heart valves

\section{RESULTS}

The cohort included 138876 adults with prosthetic heart valves (285 034 person years); 69303 (49.9\%) underwent at least one dental procedure. Among the 396615 dental procedures performed, 103463 (26.0\%) were invasive and therefore presented an indication for antibiotic prophylaxis, which was performed in 52280 (50.1\%). With a median followup of 1.7 years, 267 people developed infective endocarditis associated with oral streptococci (incidence rate 93.7 per 100000 person years, 95\% confidence interval 82.4 to 104.9). Compared with non-exposure periods, no statistically significant increased rate of oral streptococcal infective endocarditis was observed during the three months after an invasive dental procedure (relative rate 1.25 , $95 \%$ confidence interval 0.82 to $1.82 ; \mathrm{P}=0.26$ ) and after an invasive dental procedure without antibiotic prophylaxis (1.57, 0.90 to 2.53 ; $\mathrm{P}=0.08)$. In the case crossover analysis, exposure to invasive dental procedures was more frequent during case periods than during matched control periods $(5.1 \%$ v $3.2 \%$; odds ratio $1.66,95 \%$ confidence interval 1.05 to 2.63 ; $\mathrm{P}=0.03$ ).

\section{CONCLUSION}

Invasive dental procedures may contribute to the development of infective endocarditis in adults with prosthetic heart valves.

\section{Introduction}

Infective endocarditis is a rare but severe disease with an in-hospital mortality rate of about $20 \%$ and a five year mortality rate of $40 \%{ }^{1}$ This disease is also associated with high morbidity and a high cost burden, as the treatment of infective endocarditis requires prolonged hospital stay, and one out of two patients undergoes valve surgery during the acute phase of the disease. ${ }^{2}$ Strategies for antibiotic prophylaxis against infective endocarditis have been proposed for many years for patients with heart disease at risk of infective endocarditis undergoing invasive procedures responsible for bacteraemia, ${ }^{34}$ despite the lack of proof of efficacy: neither randomised clinical trials (providing the highest level of evidence) nor cohorts studies have been conducted to support an antibiotic prophylaxis strategy. Over the past three decades five case-control studies have been conducted con $^{5-9}$ only two of them established an association between dental procedures and streptococcal infective endocarditis and none of them was sufficiently powered to establish the efficacy of antibiotic prophylaxis. 
In addition to the lack of scientific evidence of efficacy for an antibiotic prophylaxis strategy, ${ }^{10}$ statistical modelling showed that "everyday low level bacteraemia" that occurs after tooth brushing, flossing, or chewing (and not covered by antibiotic prophylaxis) may more frequently trigger oral streptococcal infective endocarditis bacteraemia than invasive dental procedures. ${ }^{11-16}$ On the basis of these hypotheses, the indications for antibiotic prophylaxis have been restricted to two different strategies in all guidelines over the past decade. The 2007 US $^{17}$ and 2009/2015 European guidelines ${ }^{4}{ }^{18}$ recommend antibiotic prophylaxis only in patients with prosthetic heart valves, a history of infective endocarditis, or congenital cyanotic heart disease undergoing invasive dental procedures, whereas the UK 2008 NICE guidelines ${ }^{19}$ recommend that antibiotic prophylaxis should be withheld from all patients for all procedures.

However, a trend towards an increased incidence of infective endocarditis has been reported in some studies from the US and Germany after implementation of the 2007-09 guidelines and in the UK after the 2008 NICE guidelines, highlighting the possible role of invasive dental procedures in the development of infective endocarditis. ${ }^{20-22}$ In the UK this increased incidence, reported in 2015 in various patient groups, was observed in those with prosthetic heart valves who no longer received antibiotic prophylaxis before invasive dental procedures. Despite discordant findings from other studies ${ }^{23-25}$ these results raised the question of whether the indications for antibiotic prophylaxis may be broadened again. Although NICE guidelines were not modified immediately following this publication, the 2016 guidelines clearly specified that "antibiotic prophylaxis may be appropriate in individual cases." 26

We assessed the relation between invasive dental procedures and oral streptococcal infective endocarditis and evaluated the role of antibiotic prophylaxis, using a nationwide population based cohort and a case crossover study.

\section{Methods}

\section{Data source}

This study was based on health administrative data obtained from the French National Health Insurance general scheme covering approximately 50 million people linked with the national hospital discharge database (SNIIRAM-PMSI) by means of a unique anonymous identifier, as previously described. ${ }^{2728}$ The present study was based on data from January 2006 to December 2014 and is reported according to STROBE (statement for reporting case-control studies) guidelines. ${ }^{29}$

\section{Identification of participants}

We identified participants as those with a prosthetic heart valve using specific medical procedure codes for positioning or replacement of prosthetic heart valves (see supplementary file for list of codes) or one of the following ICD-10 (international classification of diseases, 10th revision) codes for hospital discharge diagnoses: presence of prosthetic heart valve (Z95.2), infection and inflammatory reaction due to cardiac valve prosthesis (T82.6), or mechanical complication of heart valve prosthesis (T82.0).

\section{Study design \\ Cohort study}

We carried out a nationwide population based cohort study to assess the rate of infective endocarditis associated with oral streptococci during the three months after invasive dental procedures according to different categories of exposure. This three month interval was based on data from previous studies reporting long intervals between dental procedures and the diagnosis of infective endocarditis, and data from the literature. ${ }^{30} 31$

Participants entered the cohort six months after the date of first identification of a prosthetic heart valve during the study period to ensure analysis under stable conditions. To be included in the cohort, patients had to be aged 18 years or older with no discharge diagnosis code for oral streptococcal infective endocarditis or other specific codes related to prosthetic heart valve before inclusion in the cohort (fig 1).

We followed up participants from the time of inclusion in the cohort until the study outcome (oral streptococcal infective endocarditis), or hospital admission for valve replacement, or death from any cause, or discontinuation of follow-up (defined as more than six months with no reimbursement claims for any healthcare procedures), or end of the study period (December 2014), whichever occurred first.

\section{Case crossover study}

To control for potential residual confounders, we also carried out a case crossover study, in which participants with a prosthetic heart valve and oral streptococcal infective endocarditis (ie, cases) served as their own controls during a preceding period. In this study, we selected all participants with prosthetic heart valve, as previously defined, admitted to hospital for the first time with a discharge diagnosis of oral streptococcal infective endocarditis between January 2009 and December 2014. These participants constituted the case population whether or not they were included in the cohort study. For each case we compared the presence or absence of invasive dental procedures during the three months immediately preceding oral streptococcal infective endocarditis (case period) with the presence or absence of invasive dental procedures during earlier control periods (months 9 to 7 before, months 15 to 13 before, and months 21 to 19 before) in the same participants. This method implicitly takes into account all measured and unmeasured time independent confounding factors (including oral hygiene) and can be used to investigate associations between transient exposures and acute outcome events. $^{32}$

To avoid carryover effects we separated case and controls periods from each other by a three month 


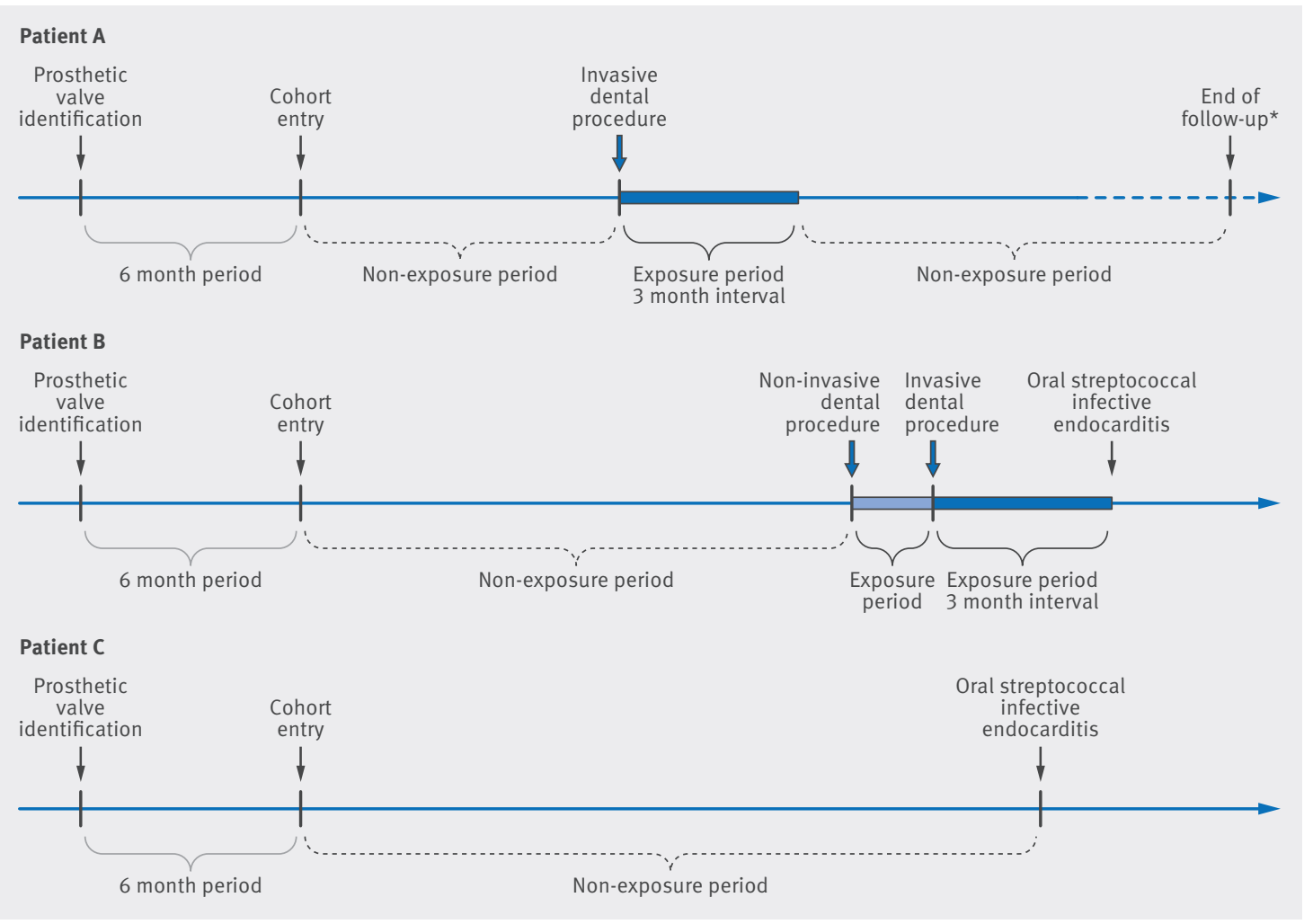

Fig 1 | Time frame of cohort study with examples of exposure during follow-up for three patients. *Death from any cause (other than oral streptococcal infective endocarditis), or admission to hospital for prosthetic heart valve or replacement of prosthetic heart valve, or lost to follow-up, or follow-up until December 2014. Participant A was exposed to an invasive dental procedure during follow-up, but did not present with oral streptococcal infective endocarditis. Participant $B$ was exposed to both a non-invasive and an invasive dental procedure during follow-up and presented with oral streptococcal infective endocarditis; during the co-exposure period, priority was given to exposure of an invasive dental procedure. Participant $\mathrm{C}$ was not exposed to any dental procedure during follow-up and presented with an oral streptococcal infective endocarditis

washout period. To be included in the case crossover study, cases had to have been followed for at least nine months before onset of oral streptococcal infective endocarditis (to allow the analysis of at least one case and one control period separated by a washout period). Figure 2 summarises the study design.

\section{Identification of dental procedures}

For each participant we identified dental procedures in the SNIIRAM database. According to the respective codes (see list in supplementary material), dental procedures were classified as invasive when they required manipulation of the gingival or periapical region of the teeth or perforation of the oral mucosa (excluding local anaesthetic injection), as recommended by 2015 European guidelines; these invasive procedures require antibiotic prophylaxis. Other dental procedures were classified as non-invasive. Two dentists, blinded to study results, determined the correspondence between codes and classification.

\section{Identification of antibiotic prophylaxis}

As antibiotic prophylaxis before invasive dental procedure was not recorded in the SNIIRAM database, we considered antibiotic prophylaxis preceding the dental procedure when the participants had an antibiotic

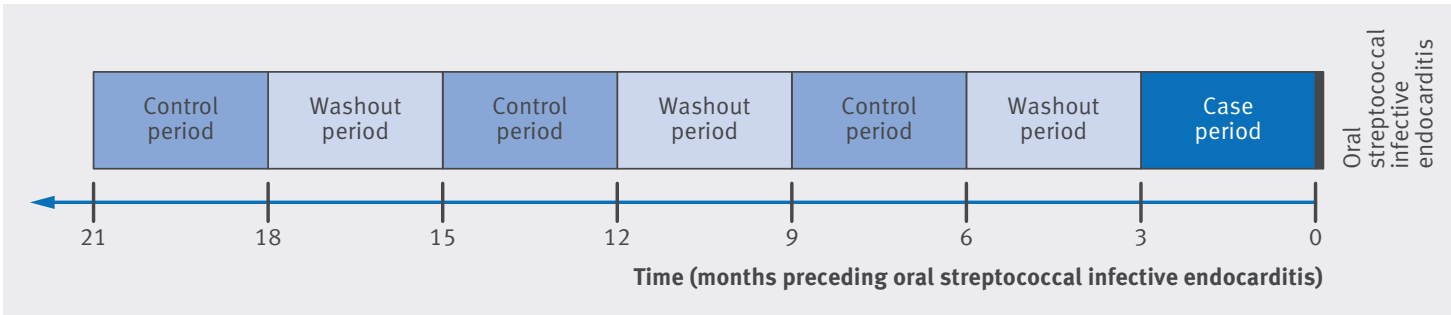

Fig 2 | Time frame of case and control periods in case crossover study, 2009-14. The case crossover design only includes cases, ie, participants who experienced the outcome events (oral streptococcal infective endocarditis), and each case acted as his or her own control 
treatment (see supplementary file for list) active against oral streptococci dispensed by pharmacists in the 21 days before the dental procedure, irrespective of whether the procedure was invasive or non-invasive. This 21 day interval was based on a previous survey conducted among 585 dentists, which showed that $84 \%$ of practitioners prescribed antibiotic treatment during the two weeks before the dental procedure. ${ }^{33}$

\section{Definition of exposure}

Initially we classified exposure into two categories according to whether the dental procedure was invasive or non-invasive. When both types of procedure were reported for the same period for a given participant, we prioritised the invasive procedure.

We then subdivided these periods into two categories according to the use of antibiotic prophylaxis, resulting in four categories: invasive dental procedure with antibiotic prophylaxis, invasive procedure without antibiotic prophylaxis, non-invasive procedure with antibiotic prophylaxis, and non-invasive procedure without antibiotic prophylaxis.

\section{Definition of oral streptococcal infective endocarditis}

We defined oral streptococcal infective endocarditis as the first hospital admission between January 2009 and December 2014 with a primary discharge diagnosis of infective endocarditis using ICD-10 codes (I33.0 for acute and subacute infective endocarditis, I33.9 for acute and subacute endocarditis, unspecified) combined with at least one secondary discharge diagnosis of streptococcal infection due to non-A, non-B, non-pneumococcal streptococcus (ICD-10 codes A40.8, A40.9, A49.1, B95.4, B95.5) as performed in a previous study. ${ }^{34}$ This algorithm was used because no codes are available for oral streptococci. We excluded patients admitted to hospital with a primary discharge diagnosis of infective endocarditis but with no identified microorganism.

To evaluate the sensitivity and positive predictive value of this algorithm for identification of cases with oral streptococcal infective endocarditis, two of the authors (ST, XD) reviewed the medical records of 130 patients admitted to a tertiary care hospital between 2008 and 2014 with at least one positive blood culture result for oral streptococci.

\section{Confounding variables}

In the SNIIRAM database we identified potential baseline confounding factors known to be related to infective endocarditis: in the year before cohort entry the presence of an implantable cardioverter defibrillator or pacemaker, intravenous drug use, dialysis dependence, and diabetes (see supplementary file for codes).

\section{Statistical analysis}

For the cohort analysis, we calculated the crude incidence rates (number of outcomes divided by cumulative person years) of oral streptococcal infective endocarditis per 100000 person years for the different exposure categories. Poisson regression was used to calculate the adjusted relative rate estimates of oral streptococcal infective endocarditis for the different categories of exposure. We used two models successively: the first model (the dental procedures model) used the two exposure categories, and the second model (the antibiotic prophylaxis dental procedures model) used the four exposure categories according to antibiotic prophylaxis use. Both models were adjusted for all potential baseline confounding factors.

For the case crossover analysis, we used conditional logistic regression to estimate the odds ratio during the case period compared with the matched control periods. We used two models according to the different exposure categories (two or four). As the temporal trend of dental procedures remained stable throughout the control periods, we did not perform any case-time control analyses.

For all analyses we considered non-exposure to be the reference group. Interaction tests using the formula of Altman and Bland ${ }^{35}$ were calculated to estimate the interaction effect between invasive and non-invasive dental procedures; we considered $\mathrm{P}<0.05$ to be statistically significant. Statistical analyses were performed with SAS 9.4 software (SAS Institute, Cary, $\mathrm{NC})$.

\section{Patient involvement}

No patients were involved in setting the research question or the outcome measures, nor were they involved in developing plans for recruitment, design, or implementation of the study. No patients were asked to advise on interpretation or writing up of results. There are no plans to provide study participants or the relevant patient community with the results of this study.

\section{Results \\ Cohort study}

\section{Participant characteristics}

The cohort comprised 138876 people with prosthetic heart valves, with 82217 (59.2\%) men and a median age of 74 years (interquartile range 63-80 years; fig 3 ). Table 1 presents the baseline characteristics of the cohort.

Incidence of oral streptococcal infective endocarditis and exposure to dental procedures

Participants were followed up for a median of 1.7 years (interquartile range 0.6-3.2 years). Among the 285034 person years, 267 cases of oral streptococcal infective endocarditis were reported, corresponding to an overall crude incidence rate of 93.7 cases per 100000 person years (95\% confidence interval 82.4 to 104.9 cases per 100000 person years).

Among the 138876 participants, 69303 (49.9\%) had at least one dental visit with an invasive or noninvasive dental procedure, with a median of 2.0 visits per participant per year (interquartile range 0.8-5.6 visits per participant per year). Overall, during the 


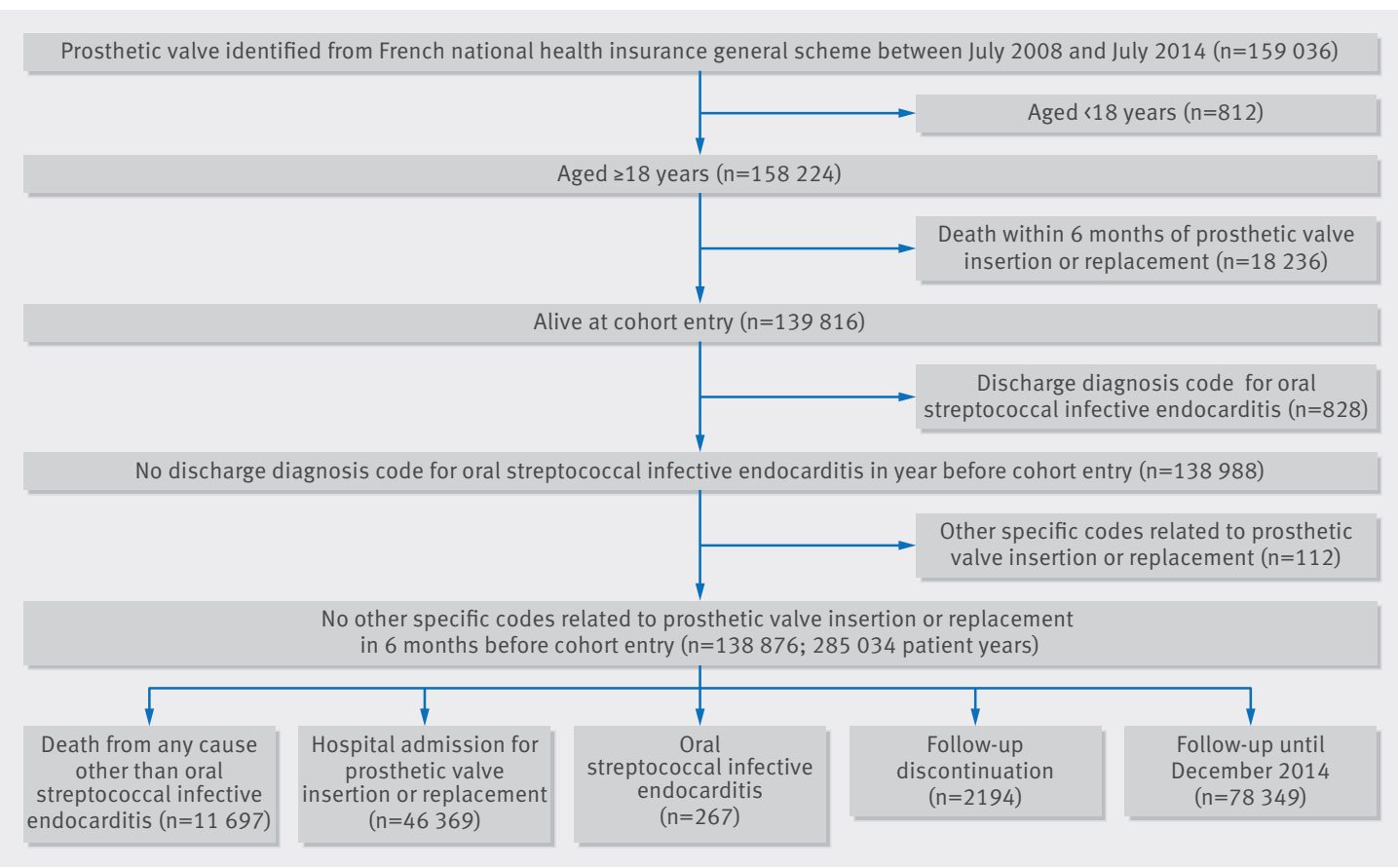

Fig 3 | Flow chart of cohort study, 2008-14. Participants entered the cohort six months after the date of first identification of the presence of a prosthetic valve during the study period to be analysed in a stable condition. At cohort entry, participants had to be aged more than 18 years without a discharge diagnosis code for oral streptococcal infective endocarditis in the previous year or other specific codes related to a prosthetic heart valve during the six months preceding cohort entry

follow-up period, 396615 dental procedures were performed in the 138876 participants, 103463 $(26.0 \%)$ of which were classified as invasive procedures requiring antibiotic prophylaxis (table 2). During the preceding 21 days, antibiotic prophylaxis was dispensed in $52280(50.1 \%)$ of these invasive procedures. The cumulative exposure period to dental procedures corresponded to 36490 person years and the non-exposure period to 248544 person years.

During the period of non-exposure to dental procedures, the incidence rate of oral streptococcal infective endocarditis was 94.6 per 100000 person years (95\% confidence interval 82.5 to 106.6 per 100000 person years). Irrespective of antibiotic

\begin{tabular}{|c|c|}
\hline Characteristics & No $(\%)$ of total population $(n=138876)$ \\
\hline Female & $56659(40.8)$ \\
\hline Male & $82217(59.2)$ \\
\hline \multicolumn{2}{|l|}{ Age (years): } \\
\hline$<30$ & $1343(1.0)$ \\
\hline $30-59$ & 23534 (17.0) \\
\hline $60-69$ & 29251 (21.1) \\
\hline $70-79$ & $45176(32.5)$ \\
\hline$\geq 80$ & $39572(28.5)$ \\
\hline ICD or pacemaker & $15643(11.3)$ \\
\hline Diabetes & $34148(24.6)$ \\
\hline Intravenous drug use & $582(0.4)$ \\
\hline Dialysis dependent & $1459(1.1)$ \\
\hline
\end{tabular}

prophylaxis, the incidence of oral streptococcal infective endocarditis varied from 72.9 per 100000 person years (39.2 to 106.6) during the three months after a non-invasive dental procedure to 118.5 per 100000 person years (56.4 to 180.6 ) during the three months after an invasive dental procedure. The overall rate of oral streptococcal infective endocarditis was 1.4 cases per 10000 invasive dental procedures.

When we subdivided exposure periods according to the use of antibiotic prophylaxis, incidence rates of oral streptococcal infective endocarditis varied from 78.1 per 100000 person years (1.6 to 154.6) during the three months after an invasive dental procedure with antibiotic prophylaxis to 149.5 per 100000 person years ( 56.8 to 242.2 ) during the three months after an invasive dental procedure without antibiotic prophylaxis (table 2).

\section{Association between dental procedures and oral streptococcal infective endocarditis}

Table 2 shows the crude incidence rate ratios of oral streptococcal infective endocarditis for the different exposure categories compared with the non-exposure period.

In the dental procedures model, after adjustment for potential baseline confounding factors (sex, age, presence of an implantable cardioverter defibrillator or pacemaker, diabetes, intravenous drug use, dialysis dependent), the rate of oral streptococcal infective endocarditis during the three months after an invasive dental procedure was not significantly different 


\begin{tabular}{|c|c|c|c|c|c|c|c|c|}
\hline Variables & $\begin{array}{l}\text { No of } \\
\text { participants }\end{array}$ & $\begin{array}{l}\text { No of } \\
\text { procedures }\end{array}$ & $\begin{array}{l}\text { Person } \\
\text { years }\end{array}$ & $\begin{array}{l}\text { No of cases } \\
\text { of oral } \\
\text { streptococcal } \\
\text { IE }\end{array}$ & $\begin{array}{l}\text { Crude incidence } \\
\text { rate of } \\
\text { oral streptococcal IE } \\
(95 \% \mathrm{CI})\end{array}$ & $\begin{array}{l}\text { Crude incidence rate } \\
\text { ratio }(95 \% \mathrm{Cl})\end{array}$ & $\begin{array}{l}\text { Adjusted relative } \\
\text { rate }^{\star}(95 \% \mathrm{Cl})\end{array}$ & $\begin{array}{l}P \\
\text { value }\end{array}$ \\
\hline Non-exposed & 138846 & & 248544 & 235 & $94.6(82.5$ to 106.6$)$ & 1.00 & 1.00 & \\
\hline \multicolumn{9}{|c|}{$\begin{array}{l}\text { Invasive dental procedure } \\
\text { period: }\end{array}$} \\
\hline Total & 33181 & 103463 & 11811 & 14 & $\begin{array}{l}118.5 \\
(56.4 \text { to } 180.6)\end{array}$ & 1.25 (0.73 to 2.00$)$ & $1.25(0.82$ to 1.82$) \dagger$ & 0.26 \\
\hline $\begin{array}{l}\text { Without antibiotic } \\
\text { prophylaxis }\end{array}$ & 21471 & 51183 & 6688 & 10 & $\begin{array}{l}149.5 \\
\text { (56.8 to 242.2) }\end{array}$ & $1.58(0.76$ to 2.87$)$ & 1.57 (0.90 to 2.53$)$ & 0.08 \\
\hline $\begin{array}{l}\text { With antibiotic } \\
\text { prophylaxis }\end{array}$ & 18863 & 52280 & 5123 & 4 & 78.1 (1.6 to 154.6) & 0.83 (0.24 to 1.99) & 0.83 (0.33 to 1.69) & 0.65 \\
\hline \multicolumn{9}{|l|}{$\begin{array}{l}\text { Non-invasive dental } \\
\text { procedure period: }\end{array}$} \\
\hline Total & 53443 & 293152 & 24679 & 18 & 72.9 (39.2 to 106.6$)$ & 0.77 (0.48 to 1.18$)$ & $0.80(0.56$ to 1.12$) \dagger$ & 0.22 \\
\hline $\begin{array}{l}\text { Without antibiotic } \\
\text { prophylaxis }\end{array}$ & 47829 & 217767 & 20131 & 13 & $64.6(29.5$ to 99.7$)$ & $0.68(0.36$ to 1.16$)$ & 0.70 (0.43 to 1.08$)$ & 0.13 \\
\hline $\begin{array}{l}\text { With antibiotic } \\
\text { prophylaxis }\end{array}$ & 19428 & 75385 & 4548 & 5 & $\begin{array}{l}109.9 \\
\text { (13.6 to 206.3) }\end{array}$ & $1.16(0.40$ to 2.59$)$ & $1.27(0.56$ to 2.42$)$ & 0.51 \\
\hline
\end{tabular}

$\mathrm{IE}=$ infective endocarditis.

*Adjusted for sex, age, presence of implantable cardioverter defibrillator or pacemaker, diabetes, intravenous drug use, dialysis dependence.

tInteraction test between invasive and non-invasive dental procedures z score=1.80 (0.485/0.270; P=0.07).

compared with the non-exposure period (fully adjusted relative rate $1.25,95 \%$ confidence interval 0.82 to 1.82; $\mathrm{P}=0.26)$; the difference between invasive and non-invasive dental procedures was not statistically significant $(\mathrm{P}=0.07$ for interaction; table 2$)$.

In the antibiotic prophylaxis dental procedures model, no statistically significant difference was observed for the rate of oral streptococcal infective endocarditis after an invasive dental procedure without antibiotic prophylaxis compared with the nonexposure period (fully adjusted relative rate 1.57, 0.90 to $2.53 ; \mathrm{P}=0.08)$.

\section{Case crossover study}

Of the 2011 participants admitted to hospital for the first time with a primary discharge diagnosis of infective endocarditis during the study period, 648 were included in the case crossover study

\begin{tabular}{|c|c|}
\hline Periods & No (\%) \\
\hline \multicolumn{2}{|l|}{ Case period $(n=648)$} \\
\hline Non-exposure & $554(85.5)$ \\
\hline Invasive dental procedure: & $33(5.1)$ \\
\hline Without antibiotic prophylaxis & $14(2.2)$ \\
\hline With antibiotic prophylaxis & $19(2.9)$ \\
\hline Non-invasive dental procedure: & $61(9.4)$ \\
\hline Without antibiotic prophylaxis & $51(7.9)$ \\
\hline With antibiotic prophylaxis & $10(1.5)$ \\
\hline \multicolumn{2}{|l|}{ Control periods $(n=1737)^{\star}$} \\
\hline Non-exposure & $1507(86.7)$ \\
\hline Invasive dental procedure: & $55(3.2)$ \\
\hline Without antibiotic prophylaxis & $25(1.4)$ \\
\hline With antibiotic prophylaxis & $30(1.7)$ \\
\hline Non-invasive dental procedure: & $175(10.1)$ \\
\hline Without antibiotic prophylaxis & $144(8.3)$ \\
\hline With antibiotic prophylaxis & $31(1.8)$ \\
\hline
\end{tabular}

with oral streptococcal infective endocarditis (see supplementary figure S1), with $69.7 \%$ of men and a median age of 77 (interquartile range 68-82) years.

\section{Exposure to dental procedures}

Among these 648 cases, 94 (14.5\%) had at least one dental procedure during the three months preceding oral streptococcal infective endocarditis: 61 (9.4\%) of them had undergone a non-invasive procedure and 33 (5.1\%) an invasive procedure. An effective antibiotic treatment was dispensed during the preceding 21 days for $19(57.6 \%)$ of the 33 participants who had undergone an invasive dental procedure (table 3 ).

The overall frequency of dental procedures was $13.3 \%$ during the control periods, with $10.1 \%$ of noninvasive procedures and $3.2 \%$ of invasive procedures. Supplementary table S1 shows the distribution of concordant and discordant matched pairs for the presence or absence of dental procedures during the case and control periods. One of the key assumptions of this approach was verified by confirming that there was no evidence of an exposure time trend during the 21 months before the event date (see supplementary figure S2).

\section{Association between dental procedures and oral streptococcal infective endocarditis}

Overall, in the dental procedures model exposure to invasive procedures was more common during case periods than during the matched control periods $(5.1 \%$ $v 3.2 \%$; odds ratio $1.66,95 \%$ confidence interval 1.05 to $2.63 ; \mathrm{P}=0.03)$, whereas exposure to non-invasive procedures was not $(9.4 \%$ v $10.1 \% ; 0.98,0.70$ to 1.36 ; $\mathrm{P}=0.16)$; the difference between invasive and noninvasive procedures was not significant $(\mathrm{P}=0.07$ for interaction; table 4).

In the antibiotic prophylaxis dental procedures model, no statistically significant differences in odds ratios 


\begin{tabular}{|c|c|c|}
\hline Dental procedures & Odds ratio $(95 \% \mathrm{Cl})$ & $P$ value \\
\hline Dental procedures model & & \\
\hline Invasive dental procedures & $1.66^{*}(1.05$ to 2.63$)$ & 0.03 \\
\hline Non-invasive dental procedures & $0.98 *(0.70$ to 1.36$)$ & 0.16 \\
\hline Invasive dental procedures: & & \\
\hline Without antibiotic prophylaxis & $1.62(0.81$ to 3.27$)$ & 0.32 \\
\hline With antibiotic prophylaxis & $1.69(0.93$ to 3.06$)$ & 0.19 \\
\hline Non-invasive procedures: & & \\
\hline Without antibiotic prophylaxis & 0.99 (0.69 to 1.42$)$ & 0.29 \\
\hline
\end{tabular}

were observed between case periods and matched control periods for any exposure category (table 4).

\section{Validation of ICD-10 coding for oral streptococcal infective endocarditis}

The study definition of oral streptococcal infective endocarditis validated using medical records of 130 participants admitted to hospital with at least one positive blood culture result for oral streptococci had a sensitivity of $54 \%$ and a positive predictive value of $100 \%$.

\section{Discussion}

This study, combining analyses of a large cohort of patients with prosthetic heart valves and of a case crossover study, shows that invasive dental procedures may contribute to the development of infective endocarditis in this population of patients known to present a high incidence of this disease and to be at high risk of morbidity and mortality associated with the disease. However, a statistically significant reduction in the rate of developing infective endocarditis after such procedures was not observed among those participants receiving antibiotic prophylaxis.

The cohort was large, comprising 138876 participants with prosthetic heart valves, which represents approximately two thirds of the corresponding French population based on our previous estimations. ${ }^{36}$ The availability of a large cohort is of particular importance in the study of infective endocarditis in view of the low incidence of this disease. External comparison is difficult because no similar information is available from other sources or other countries. Given the selection process, there is no reason to believe that this study population is not representative of the entire population with prosthetic heart valves; the results of this study can therefore be extrapolated to the larger population.

The analysis of the cohort provides original data in this population of participants with prosthetic heart valves, with high exposure to dental procedures (396615 procedures) but which varied considerably from one participant to another, as only half of the cohort received dental care during the mean followup of 1.7 years, despite the recommendation to visit a dentist twice yearly.
In addition, only one half of invasive procedures (one quarter of all dental procedures) were associated with antibiotic prophylaxis, despite recommendations. This is a low proportion but consistent with other reports in France. ${ }^{9}$

In this cohort of participants with prosthetic heart valves, the incidence of infective endocarditis, regardless of the microorganism (840 cases per 100000 person years, data not shown) and the risk of developing oral streptococcal infective endocarditis after an invasive dental procedure (1.4 out of 10000 ), were both in the same range as the incidence rates reported in the literature. ${ }^{36}$

Interestingly, for the first time in this population the cohort provides an estimate for the incidence of oral streptococcal infective endocarditis in relation to everyday life bacteraemia (ie, oral hygiene habits such as tooth brushing, use of toothpicks, flossing, or chewing) during non-exposure periods. The microtrauma caused by these everyday activities has been identified to induce oral streptococcal bacteraemia, in fairly similar proportions to those of invasive oral procedures for which antibiotic prophylaxis is recommended. ${ }^{37}$ The fact that the cumulative non-exposure periods were much longer than the exposure periods (248 544 person years $v 36490$ person years, ie, seven times higher) strongly suggests that most cases of infective endocarditis are due to everyday life bacteraemia, which is consistent with the results of the case crossover study, reporting a history of dental procedures in the three months preceding infective endocarditis in only a small number of participants.

This cohort shows that the incidence of oral streptococcal infective endocarditis during the three months after an invasive dental procedure was higher, but not statistically significantly higher, in participants not receiving the recommended antibiotic prophylaxis. However, the analysis performed in this cohort is based on pooled data derived from a heterogeneous population of patients in terms of use of dental care (measured in our database) and certainly in terms of oral hygiene and dental status (not measured in our database), which represent a potential source of bias ${ }^{9}$ in the non-exposure periods.

The case crossover study may ensure better control for these confounding factors (individual oral hygiene 
and dental status), as each participant constituted his or her own control. The case crossover analysis including 648 participants with oral streptococcal infective endocarditis showed a statistically significant association between invasive dental procedures and oral streptococcal infective endocarditis. Using the same case crossover design in 170 participants with infective endocarditis, one study did not observe any statistically significant difference in the number and type of dental procedures performed during the three month period before admission for infective endocarditis compared with control periods. ${ }^{38}$ However, only 49 viridans streptococci were analysed and 44\% of participants had a prosthetic heart valve. Similarly, in a recent case crossover study based on Taiwan's national health insurance programme, one study reported that dental procedures did not increase the risk of infective endocarditis. ${ }^{31}$ However, only 125 patients with infective endocarditis with valvular heart disease (proportion of patients with prosthetic heart valves not specified) were included in the analysis and no data were available concerning the microorganisms responsible for infective endocarditis. The small numbers of cases with streptococcal infective endocarditis in these two studies limited the statistical power of the analyses.

In our case crossover analysis we found a low rate of invasive dental procedures during the three months preceding oral streptococcal infective endocarditis (5.1\%, but consistent with the literature) showing that most cases of streptococcal infective endocarditis were not associated with recent dental procedures. Notably, this case crossover analysis was not affected by recall bias, as dental procedures were retrieved from the SNIIRAM database. In a recently published case-control study performed in patients with infective endocarditis admitted to hospital in six French tertiary care hospitals (regardless of predisposing heart conditions), we showed that $16.9 \%$ of participants with definite streptococcal infective endocarditis had undergone dental procedures during the preceding three months. ${ }^{9}$

Although the case crossover analysis revealed a statistically significant association between invasive dental procedures and oral streptococcal infective endocarditis among participants with prosthetic heart valves, there is no good evidence to support a different effect between invasive and non-invasive dental procedures probably as a result of lack of power $(\mathrm{P}=0.07)$. We failed to show the efficacy of antibiotic prophylaxis, again possibly owing to the lack of power attributable to the small number of participants in the different subgroups included in the antibiotic prophylaxis dental procedures model, in which only discordant pairs contributed.

\section{Limitations of this study}

Both the cohort and the case crossover study present several limitations. Firstly, they are subject to the limitations related to the use of administrative databases, which may include inaccurate coding of the participant's clinical diagnosis and procedures, with clinical information limited to conditions and treatments defined by ICD-10 codes. Secondly, identification of the causal microorganism was also subject to limitations, as no specific ICD-10 codes are available to identify oral streptococci, and the diagnosis of infective endocarditis was not confirmed by using modified Duke criteria. However, ICD-10 codes were validated by referring to clinical records in selected participants, which showed high positive predictive values for the codes used. Finally, no information is available on adherence to antibiotic treatment after dispensing by the pharmacy.

\section{Conclusion}

Results of our two studies differed in terms of the statistical significance for the association between invasive dental procedures and oral streptococcal infective endocarditis. However, both indicated the same direction of effect, suggesting that invasive dental procedures may be associated with oral streptococcal infective endocarditis, although the magnitude of this association remains uncertain. Further studies based on an even larger scale, requiring international collaborations are needed to provide a sufficient statistical power to confirm these results and to evaluate the role of antibiotic prophylaxis, pending the initiation of a randomised clinical trial. ${ }^{39}$

Contributors: XD, FA, and BH had the idea for the study. ST, POB, AW, FA, JR, and XD conceived the study design. POB and ST planned the study and ST drafted the manuscript. POB, ST, PL, and SM performed data management. ST and POB did the statistical analyses. FA and XD were responsible for management of the project and oversaw the study. All authors contributed to the interpretation of the data, revised the manuscript, and approved the final manuscript. XD, FA, and ST are the guarantors.

Funding: This research was funded by the French National Health Insurance Fund (CNAMTS) and Université Paris Diderot, France. The present paper represents the opinions of the authors and does not necessarily reflect the position of their employers.

Competing interests: All authors have completed the ICMJE uniform disclosure form at www.icmje.org/coi_disclosure.pdf and declare: no support from any organisation for the submitted work; no financial relationships with any organisations that might have an interest in the submitted work in the previous three years; no other relationships or activities that could appear to have influenced the submitted work.

Ethical approval: This study was approved by the French data protection agency Commission Nationale de l'Informatique et des Libertés (regulatory decision DE-2011-078).

Data sharing: No additional data available.

Transparency: The manuscript's guarantors (XD, FA, and ST) affirm that this manuscript is an honest, accurate and transparent account of the study being reported; that no important aspects of the study have been omitted; and that any discrepancies from the study as planned (and, if relevant, registered) have been explained.

This is an Open Access article distributed in accordance with the Creative Commons Attribution Non Commercial (CC BY-NC 4.0) license, which permits others to distribute, remix, adapt, build upon this work non-commercially, and license their derivative works on different terms, provided the original work is properly cited and the use is noncommercial. See: http://creativecommons.org/licenses/by-nc/4.0/.

1 Hoen B, Duval X. Clinical practice. Infective endocarditis. N Engl J Med 2013;368:1425-33. doi:10.1056/NEJMcp1206782.

2 Verhagen DWM, Hermanides J, Korevaar JC. Health-related quality of life and posttraumatic stress disorder among survivors of leftsided native valve endocarditis. Clin Infect Dis 2009;48:1559-65. doi:10.1086/598930.

3 Baddour LM. Prophylaxis of infective endocarditis: prevention of the perfect storm. Int J Antimicrob Agents 2007;30(Suppl 1):S37-41. doi:10.1016/j.ijantimicag.2007.06.033.

4 Habib G, Lancellotti P, Antunes M), el al. 2015 ESC Guidelines for the management of infective endocarditis: The Task Force for the Management of Infective Endocarditis of the Europe an Society of Cardiology (ESC). Endorsed by: European Association for Cardio-Thoracic Surgery (EACTS), the European Association of Nuclear Medicine (EANM). Eur Heart/ 2015;36:3075-128. doi:10.1093/eurheartj/ehv319. 
5 Imperiale TF, Horwitz RI. Does prophylaxis prevent postdental infective endocarditis? A controlled evaluation of protective efficacy. Am J Med 1990;88:131-6. doi:10.1016/0002-9343(90)90461-L

6 Van der Meer JT, Van Wijk W, Thompson J, Vandenbroucke JP, Valkenburg HA, Michel MF. Efficacy of antibiotic prophylaxis for prevention of native-valve endocarditis. Lancet 1992:339:135-9. doi:10.1016/0140-6736(92)90207-

7 Lacassin F, Hoen B, Leport C. Procedures associated with infective endocarditis in adults. A case control study. Eur Heart / 1995;16:1968-74. doi:10.1093/oxfordjournals.eurheartj. a060855

8 Strom BL Abrutyn E Berlin IA. Dental and cardiac risk factors for infective endocarditis. A population-based, case-control study. Ann Intern Med 1998;129:761-9. doi:10.7326/0003-4819-129-10199811150-00002

9 Duval X, Millot S, Chirouze CE, el al. Oral Streptococcal Endocarditis, Oral Hygiene Habits, and Recent Dental Procedures: A Case-Contro Study. Clin Infect Dis 2017;64:1678-85. doi:10.1093/cid/cix237.

10 Cahill TJ, Harrison JL, Jewell P. Antibiotic prophylaxis for infective endocarditis: a systematic review and meta-analysis. Heart 2017;103:937-44. doi:10.1136/heartjnl-2015-309102.

11 Forner L, Larsen T, Kilian M, Holmstrup P. Incidence of bacteremia after chewing, tooth brushing and scaling in individuals with periodontal inflammation. J Clin Periodontol 2006;33:401-7. doi:10.1111/j.1600-051X.2006.00924X.

12 Roberts GJ. Dentists are innocent! "Everyday" bacteremia is the real culprit: a review and assessment of the evidence that dental surgical procedures are a principal cause of bacterial endocarditis in children. Pediatr Cardiol 1999;20:317-25. doi:10.1007/ s002469900477.

13 Durack DT. Prevention of infective endocarditis. N Engl J Med 1995:332:38-44. doi:10.1056/NEIM199501053320107.

14 Duval X, Leport C. Prophylaxis of infective endocarditis: current tendencies, continuing controversies. Lancet Infect Dis 2008;8: 225-32. doi:10.1016/S1473-3099(08)70064-1.

15 Glenny A-M, Oliver R, Roberts GJ, Hooper L, Worthington HV. Antibiotics for the prophylaxis of bacterial endocarditis in dentistry. Cochrane Database Syst Rev 2013;CD003813:CD003813. doi:10.1002/14651858.CD003813.pub4

16 Veloso TR, Amiguet M, Rousson V. Induction of experimental endocarditis by continuous low-grade bacteremia mimicking spontaneous bacteremia in humans. Infect Immun 2011;79: 2006-11. doi:10.1128/IAl.01208-10.

17 Wilson W, Taubert KA, Gewitz M, el al. Prevention of infective endocarditis: guidelines from the American Heart Association: a guideline from the American Heart Association Rheumatic Fever, Endocarditis, and Kawasaki Disease Committee, Council on Cardiovascular Disease in the Young, and the Council on Clinical Cardiology, Council on Cardiovascular Surgery and Anesthesia, and the Quality of Care and Outcomes Research Interdisciplinary Working Group. Circulation 2007;116:1736-54. doi:10.1161/ CIRCULATIONAHA.106.183095.

18 Habib G, Hoen B, Tornos P, el al. Guidelines on the prevention, diagnosis, and treatment of infective endocarditis (new version 2009): the Task Force on the Prevention, Diagnosis, and Treatment of Infective Endocarditis of the European Society of Cardiology (ESC). Eur Heart J 2009;30:2369-413. doi:10.1093/eurheartj/ehp285.

19 Richey R, Wray D, Stokes T; Guideline Development Group. Prophylaxis against infective endocarditis: summary of NICE guidance. BMJ 2008;336:770-1. doi:10.1136/bmj.39510.423148. $\mathrm{AD}$

20 Keller K, von Bardeleben RS, Ostad MA. Temporal Trends in the Prevalence of Infective Endocarditis in Germany Between 2005 and 2014. Am J Cardiol 2017;119:317-22. doi:10.1016/j. amjcard.2016.09.035.

21 Pant S, Patel NJ, Deshmukh A. Trends in infective endocarditis incidence, microbiology, and valve replacement in the United States from 2000 to 2011. J Am Coll Cardiol 2015;65:2070-6. doi:10.1016/i.jacc.2015.03.518.

22 Dayer MJ, Jones S, Prendergast B, Baddour LM, Lockhart PB, Thornhill MH. Incidence of infective endocarditis in England 2000-13: a secular trend, interrupted time-series analysis. Lancet 2015:385:1219-28 doi:10.1016/S01406736(14)62007-9.
23 Desimone DC, Tleyjeh IM, Correa de Sa DD, el al. Incidence of infective endocarditis caused by viridans group streptococci before and after publication of the 2007 American Heart Association's endocarditis prevention guidelines. Circulation 2012;126:60-4 doi:10.1161/CIRCULATIONAHA.112.095281.

24 Duval X, Delahaye F, Alla F, el al. Temporal trends in infective endocarditis in the context of prophylaxis guideline modifications: three successive population-based surveys. J Am Coll Cardiol 2012:59:1968-76. doi:10.1016/j.jacc.2012.02.029.

25 Bikdeli B, Wang Y, Kim N, Desai MM, Quagliarello V, Krumholz HM. Trends in hospitalization rates and outcomes of endocarditis among Medicare beneficiaries. J Am Coll Cardiol 2013;62:2217-26. doi:10.1016/j.jacc.2013.07.071

26 Thornhill MH, Dayer M, Lockhart PB. A change in the NICE guidelines on antibiotic prophylaxis. Br Dent/ 2016;221:112-4. doi:10.1038/ sj.bdj.2016.554.

27 Tuppin P, de Roquefeuil L, Weill A, Ricordeau P, Merlière Y. French national health insurance information system and the permanent beneficiaries sample. Rev Epidemiol Sante Publique 2010;58: 286-90. doi:10.1016/j.respe.2010.04.005.

28 Moulis G, Lapeyre-Mestre M, Palmaro A, Pugnet G, Montastruc JL, Sailler $\mathrm{L}$. French health insurance databases: What interest fo medical research?Rev Med Interne 2015;36:411-7. doi:10.1016/j. revmed.2014.11.009

29 von Elm E, Altman DG, Egger M, Pocock SJ, Gøtzsche PC, Vandenbroucke JP; STROBE Initiative. Strengthening the Reporting of Observational Studies in Epidemiology (STROBE) statement: guidelines for reporting observational studies. BMJ 2007;335:806 8. doi:10.1136/bmi.39335.541782.AD.

30 N'Guyen Y, Duval X, Revest M, el al. Time interval between infective endocarditis first symptoms and diagnosis: relationship to infective endocarditis characteristics, microorganisms and prognosis. Ann Med 2017:49:117-25. doi:10.1080/07853890.2016.1235282.

31 Chen P-C, Tung Y-C, Wu PW. Dental Procedures and the Risk of Infective Endocarditis. Medicine (Baltimore) 2015:94:e1826. doi:10.1097/MD 0000000000001826

32 Maclure M. The case-crossover design: a method for studying transient effects on the risk of acute events. Am J Epidemiol 1991;133:144-53. doi:10.1093/oxfordjournals.aje. a115853

33 Cloitre A. Antibioprophylaxie de l'endocardite infectieuse, respect des recommandations de bonnes pratiques, enquête nationale auprès des chirurgiens-dentiste. 2013. http://www.sudoc. fr/177895535 (accessed 16 Mar 2013)

34 Toyoda N, Chikwe J, Itagaki S, Gelijns AC, Adams DH, Egorova NN. Trends in Infective Endocarditis in California and New York State, 1998-2013. JAMA 2017;317:1652-60. doi:10.1001/ jama.2017.4287

35 Altman DG, Bland IM. Interaction revisited: the difference between two estimates. BMJ 2003;326:219. doi:10.1136/ bmj.326.7382.219

36 Duval X. Alla F, Hoen B. Estimated risk of endocarditis in adults with predisposing cardiac conditions undergoing dental procedures with or without antibiotic prophylaxis. Clin Infect Dis 2006;42:e102-7. doi:10.1086/504385.

37 Zhang W, Daly CG, Mitchell D, Curtis B. Incidence and magnitude of bacteraemia caused by flossing and by scaling and root planing. I Clin Periodontol 2013;40:41-52. doi:10.1111/jcpe.12029.

38 Porat Ben-Amy D, Littner M, Siegman-Igra Y. Are dental procedure an important risk factor for infective endocarditis? A case-crossover study. Eur J Clin Microbiol Infect Dis 2009;28:269-73. doi:10.1007/ s10096-008-0622-3.

39 Hoen B, Duval X. Infective endocarditis. N Engl Med 2013;369:785. doi:10.1056/NEJMc1307282.

Supplementary information: figures S1 and S2 and table S1

Supplementary information: identification of individuals with prosthetic heart valves and constitution of study population 